\title{
Absolute or Relative: What do we need to know about moisture levels in historic building conservation?
}

\author{
Tobit Curteis
}

Tobit Curteis Associates LLP, Cambridge, UK

\begin{abstract}
While much of the research into moisture and buildings is about absolute values, in building conservation projects, relative values are both of more use and practical to obtain. This paper examines why this is the case and how low precision measurements can be usefully employed in conservation diagnostics and control. Peer-review under the responsibility of the organizing committee of the ICMB21.
\end{abstract}

Keywords: moisture; measurement; thermal imaging;building conservation; electrical capacitance;microwave

\section{Introduction/Background}

Water is a key factor in the long-term deterioration of historic buildings. Sometimes damage is directly caused by water, for instance freeze thaw cycles or dissolution of a soluble fraction in stone, while sometimes the effect is indirect as with salt dissolution/crystallisation or microbiological attack. Identifying the location and level of the water affecting the structure is a critical part of controlling the deterioration process. In the air surrounding the object, measuring and understanding the behaviour of water vapour is comparatively simple. Within solid structures however, determining distribution and concentration of water is more complex. Nevertheless, without this information it is usually impossible to accurately identify the water source and whether the observed deterioration processes are associated with liquid water or water vapour.

\section{Absolute or Relative}

Much of the research that is carried out into water levels in materials focuses on identifying absolute values and it is a characteristic of the field of conservation that our technology is often borrowed from industrial applications where understanding absolute moisture content is essential. Many commonly used instruments employing electrical characteristics as a proxy for water, can be highly accurate when material type and condition is predictable, as for instance, in timber and paper.

When used on building materials, the instrument calibration is no longer relevant. This is not to say that they cannot be recalibrated for, for example, stone and brick, and some good research has been carried out in this area [1]. However, the problem is rarely the material type but rather the heterogeneous and unknown nature of the historic structures where only the surface materials and characteristics are understood with accuracy.

For most instrumental investigations, access is limited to the surface of a structure and it is extremely difficult to separate out the effects of the variation materials in depth. This is something that is observed with infrared thermography (IRT) when the proxy for water is surface temperature. However, IRT is examining IR radiation from the surface of the object which might be affected by the reflectance or emissivity of the material, as well as the thermal characteristics of materials below the surface in addition to the evaporative effect of any water content.

The same issue is encountered with the use of instruments examining electrical characteristics, such as resistance (ER), capacitance (EC) or microwaves (MW), as these are affected by many factors other than water, including material type, density and contamination with electrolytes. Although the effects vary between the different instruments, they all suffer from the same vulnerability that moisture content is displayed as a single number which might indicate that the test material has a high moisture content and few other influencing factors, or a high level of influencing factors and a low moisture content. Instruments such as microwaves, which have considerable depth of penetration, are further complicated by the fact that there is no indication of at what depth and why the signal from the instrument was attenuated. Is the test material saturated at the surface 


\section{ICMB21}

and dry after $10 \mathrm{~mm}$ or dry at the surface and saturated $100 \mathrm{~mm}$ into the structure? Or is there no water present and just an influencing material in the test material? The single figure displayed does not provide that information.

\section{Field Use}

With so many apparent weaknesses in these different indirect moisture measurement instruments, why does the building conservation field persist in their use? The answer lies in the fact that the metric which is of importance is relative moisture readings rather than absolute values. The characteristic which is generally useful is how moisture is distributed across a large and heterogeneous structure and variations in severity which, in combination, indicate the significance of the problem and likely sources of water.

To use this approach effectively, it is necessary to combine different data sets, in order to exclude some of the misleading effects which can influence a particular measurement type. A thermal imaging survey of the wall, in isolation, may produce results which include influences of liquid water, thermal bridging and air leakage. However, used in combination with an EC survey, which measures dielectric constant, and MW which measures attenuation of signals at depth, common patterns become apparent which, in combination, point to water distribution in a particular area and at a particular depth [2].
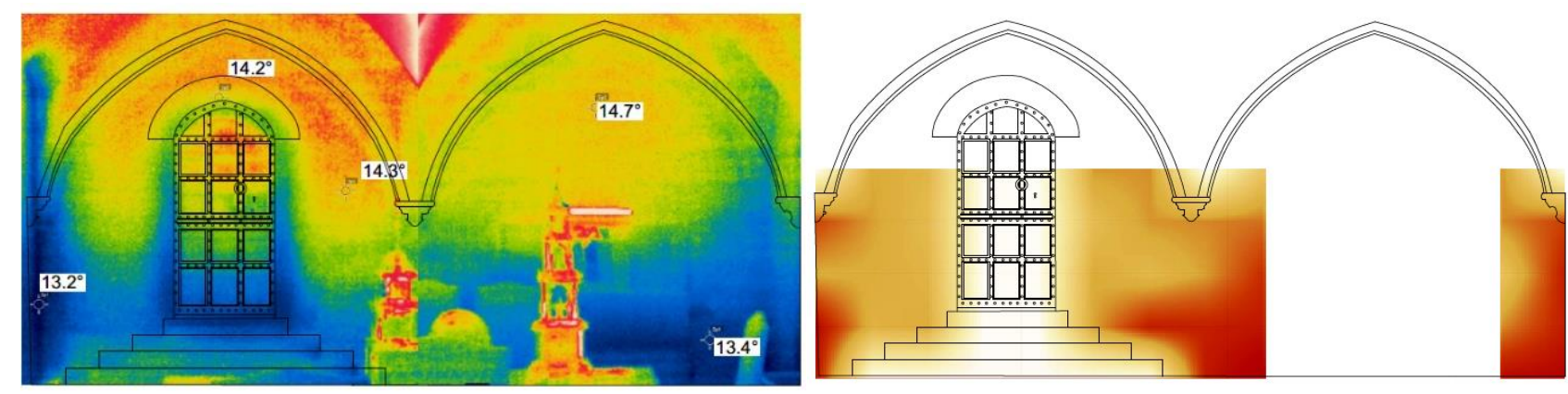

Figures $1 \& 2$. Combined thermal imaging and microwave mapping showing likely moisture patterns.

Field calibration is a prerequisite for the effective use of this type of instrument. Areas of the building should be identified where moisture conditions are predictable and might be considered "dry" and measurements can be taken to provide a calibration for this particular building. The instrument can then be used on an unpredictable area with a basic understanding of the wet/dry metric and what the numerical or visual output means.

Moisture assessment of this type also remains useful because of the speed of data capture. Analysing absolute moisture content inevitably means taking a limited number of samples and investing considerable time in their processing. By contrast, using instrumentation which allows relative measurements to be taken swiftly, an entire building structure can be examined, and patterns established in a matter of hours which, in terms of diagnostics on practical field projects, is often a far more useful tool than precision analysis.

There are cases where an understanding of absolute values may be necessary for controlling deterioration. In this case, destructive sampling, such as core sampling and gravimetric or carbide analysis, can be employed and results can also provide baseline data against which non-destructive instruments can be calibrated.

This is not to suggest that the analysis of absolute moisture values in historic building projects is not useful, and in specific applications, for instance where a precise control over salt deliquescence is required, it can be critical. However, in most cases, effective mapping of distribution patterns and levels of relative severity is a more useful and practical tool in the diagnosis and control of moisture deterioration in historic buildings.

\section{References}

[1] Julie Eklund, Hong Zhang, Heather Viles, Tobit Curteis, Using hand-held moisture meters on limestone: some factors affecting their performance and guidelines for best practice, International Journal of Architectural Heritage, (2015)

[2] Robyn Pender, Tobit Curteis, Brian Ridout, (Eds.) Building Environment; in Bill Martin and Chris Wood (Series Eds.), English Heritage Practical Building Conservation, London (2014) 